\title{
Magnetic orientation of soft particles in a jammed solid
}

\author{
Camille Dagallier, ${ }^{a}$ Frédéric Cardinaux,${ }^{a}$ Hervé Dietsch ${ }^{* b}$ and Frank Scheffold ${ }^{* a}$
}

Densely packed atoms, molecules, or small particles can get trapped in a jammed state thereby avoiding crystallization. The resulting amorphous structures display complex, spatially heterogeneous, trapping potentials in stark contrast to the uniform case of a crystal. Here we study active and passive rotational motion in a jammed colloidal dispersion of particles consisting of an anti-ferromagnetic core and a thermo-sensitive microgel soft shell. We determine the order parameter as a function of volume fraction from optical birefringence measurements. A simple model allows us to discriminate the orientation and relaxation of different subsets of particles according to their elastic trapping state.

\section{Introduction}

Sufficiently decreasing the free volume in colloidal dispersions results in a dramatic slowing down of the motion of the colloidal phase. If the system is not given enough time to organize into a stable crystalline phase it enters a jammed phase that retains the structural disorder of a liquid, and, since there is no room for reorganization, remains in this metastable state on timescales much longer than that of the experiments. ${ }^{1}$ It is generally recognized that both deformable and hard spheres are jammed into a solid state at a critical density. ${ }^{1-3}$ Although soft particles exhibit a similar behaviour to hard spheres when approaching the jamming transition from the liquid side, the compressibility becomes crucial once particles are in contact. ${ }^{4,5}$ A remarkable characteristic of soft particles is their ability to occupy space much more efficiently, allowing the dispersed phase to reach much higher volume filling than the random close packing of spheres. ${ }^{6}$

In addition to the soft particles elasticity, the properties of such jammed systems critically depend on the structure of the disordered solid. ${ }^{1}$ Since the structure is random and lacks any long-range symmetry a theoretical description is much more difficult. General geometrical arguments show that a minimum number of average contacts $Z=6$ is required to form a solid material. ${ }^{7}$ At this point the solid is marginal and the random structure is mechanically stable only for $Z>6$, for monodisperse hard spheres this happens at a volume fraction $\phi \approx 0.64 .{ }^{8}$ Above the transition the material properties scale with the mean number

${ }^{a}$ University of Fribourg, Soft Matter and Photonics Group and Fribourg Center for Nanomaterials, Department of Physics, Chemin du Musée 3, 1700 Fribourg, Switzerland. E-mail: Frank.Scheffold@unifr.ch; Fax: +4126300 9060; Tel: +41263009117

${ }^{b}$ University of Fribourg, Adolphe Merkle Institute and Fribourg Center for Nanomaterials, Route de l'Ancienne Papeterie, P.O. box 209, 1723 Marly 1, Switzerland. E-mail: Herve.Dietsch@unifr.ch; Fax: +41 26300 9624; Tel: +41263009137 of contacts. ${ }^{7}$ This number increases as the spheres are slightly compressed. For frictionless perfect spheres rotational degrees of freedom are degenerate. In contrast, for anisotropic particles, the jamming transition follows a distinctly different scenario with the addition of many zero frequency vibrations due the freezing of rotational degrees of freedom. ${ }^{9-12}$ Despite this, the volume fraction where particles jam appears to be affected only weakly by anisotropy. ${ }^{9}$ The jamming of rotational motion has also been used as a local probe to monitor the glass transition in polymeric systems under shear. ${ }^{13}$

Over the last decade a plethora of numerical, theoretical and experimental studies have addressed various aspects of this transition. ${ }^{1}$ The body of experimental work is in most cases limited to study bulk averages such as the elastic modulus or the intermediate scattering function. Unfortunately, it is impossible to trace back directly from these measurements to the highly heterogeneous structural and dynamic properties of jammed solids. Measurements on granular materials have clarified some aspects but fail to address others due to the unavoidable presence of friction and the difficulties in establishing a real temperature scale in intrinsically athermal systems. ${ }^{1,7}$ Moreover the many experiments carried out on 2D systems, for example using stress birefringence, ${ }^{7}$ can only partially be translated to the more relevant $3 \mathrm{D}$ case. Three-dimensional microscopy appears to be an obvious solution of this problem. While experiments of this kind have revealed important information about the structure of jammed solids ${ }^{14}$ they usually cannot measure interaction forces and trapping energies since thermal translational motion is restricted to the nanometre scale and external forces on individual particles are difficult to apply.

Here we study active and passive rotational motion in a jammed colloidal dispersion of particles consisting of an anti-ferromagnetic core and a thermo-sensitive soft shell. ${ }^{15-18}$ By measuring the optical birefringence we gain the ability to probe directly trapping forces of deeply jammed colloidal particles in three dimensions. We develop a simple model to distinguish the 
orientation and relaxation of different subsets of particles according to their elastic trapping state.

\section{Experiments and results}

Our particles consist of a hematite anti-ferromagnetic core coated with a silica layer, covered by a chemically grafted soft shell of cross-linked poly( $N$-isopropylacrylamide), as described previously. ${ }^{15}$ The polymer has a lower critical solution temperature (LCST) of approximately $33{ }^{\circ} \mathrm{C}$. Above the LCST, the microgel network expels water and is collapsed. Upon lowering the temperature the network is taking up water and the particles swell to about twice their original size (by radius). ${ }^{15}$ Fig. $1 \mathrm{~b}$ shows a transmission electron microscopy (TEM) image of a particle on a carbon film under high vacuum. Although the collapsed particles have an almost spherical shape (aspect ratio roughly $1.2 \pm 0.2)$ their interactions are not isotropic. Due the presence of the rigid elongated hematite core, the microgel-shell is much thicker around the particle waist compared to the tip, hence leading to an asymmetric elastic response upon deformation. The hydrodynamic radius $R_{\mathrm{h}}$ of the particles is determined by dynamic light scattering. ${ }^{15}$ For $T<T_{\mathrm{c}}$ it can be empirically represented by $R_{\mathrm{h}}=R\left(1-T / T_{\mathrm{c}}\right)^{\gamma}$, with $R=592 \mathrm{~nm}, T_{\mathrm{c}}=$ $33.7{ }^{\circ} \mathrm{C}$, and $\gamma=0.14 .{ }^{15}$ The number density is determined for a diluted sample by directly counting particles using bright field microscopy within a known volume. We prepare a dense dispersion with a particle number density $N_{\mathrm{p}}=1.2 \pm 0.02$ particles $\mu \mathrm{m}^{-3}$ in order to observe a liquid behaviour above $25^{\circ} \mathrm{C}$ and drive the formation of amorphous solids below $25^{\circ} \mathrm{C}$ with an effective volume fraction $\phi_{\text {eff }}=(4 \pi / 3) N_{\mathrm{p}} R_{\mathrm{h}}{ }^{3}$. We then vary the temperature from $30{ }^{\circ} \mathrm{C}$ to $12{ }^{\circ} \mathrm{C}$ allowing to control of the effective volume fraction over the range $0.4<\phi_{\text {eff }}<0.9$ (Fig. 1a).

Oscillatory shear measurements at $\omega=1 \mathrm{rad} \mathrm{s}^{-1}$ are conducted with a Paar Physica MCR 300 Rheometer in a cone-plate geometry, using a cone with a diameter of $5 \mathrm{~cm}$ and an angle of $1^{\circ}$. The shear modulus of the dispersion displays the hallmarks of
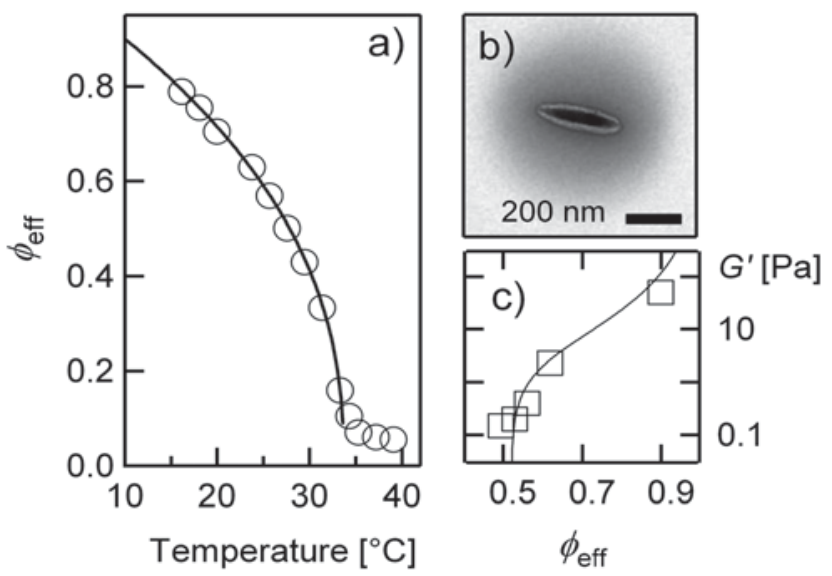

Fig. 1 Multi-responsive hematite/silica/PNiPAM particle dispersions. (a) Temperature dependence of the effective volume fraction $\phi_{\text {eff }}$ at a number density $N_{\mathrm{p}}=1.2 \pm 0.02$ particles $\mu \mathrm{m}^{-3}$. The solid line shows an empiric fit to the experimental data. (b) Transmission electron microscopy image of a hematite/silica/PNiPAM particle. (c) Elastic modulus $G^{\prime}$ as a function of effective volume fraction $\phi_{\text {eff. }}$ The line is a guide to the eye. the jamming transition of soft spheres. ${ }^{2}$ For effective volume fractions $\phi_{\text {eff }} \geq 0.55$ a finite, albeit small, elastic modulus is detected that can be attributed to entropic effects. ${ }^{2}$ For $\phi_{\text {eff }} \geq$ 0.62 particles have swollen in contact and now the modulus $G^{\prime}$ rises sharply, a clear indication for the jamming transition (Fig. 1c).

While the interparticle interactions, the rheology and the dynamics of the system are essentially set by the softness of the PNIPAM shell as well as overall shape and size of the particles, their magnetic properties are determined by the ellipsoidal paramagnetic core. In particular, the magnetic moment $\mu$ of the hematite core orients perpendicular to the long axis and its magnitude can be estimated taking into account the size of the core. ${ }^{19}$ For our particles, we obtain a value of $\mu \approx 9.2 \times 10^{-19} \mathrm{~J}$ $\mathrm{T}^{-1}$. Upon application of an external magnetic field, the long axes of the paramagnetic cores tend to align in a plane perpendicular to the field with a distribution of polar angles peaked at $\pi / 2$ (Fig. 2). This gives rise to optical birefringence with the optical axis in the co-planar direction to the long axis of the hematite cores. Therefore, the degree of orientational order can be determined by measuring the optical phase shift $\delta$ for polarized light travelling through the dispersion. Indeed, the order parameter $S$, which quantifies the degree of orientational order, depends upon the birefringence through: $S=(n \cdot \Delta n) /\left(2 \pi N_{\mathrm{p}} F\right) \propto$ $\delta$, where $n$ is the average refractive index, $\Delta n$ is the refractive index difference, and $F$ is a polarizability shape factor. ${ }^{20}$ Although this expression has been established for particles smaller than $100 \mathrm{~nm}$, it is predicted to apply also to the case of larger particles when the imposed magnetic fields are static. ${ }^{20}$

For the birefringence measurements the sample is contained within a flat capillary with an optical path of $100 \mu \mathrm{m}$, sealed with UV-curable glue, and placed on a copper sample holder. Temperate control is provided by using Peltier elements and thermal paste. A homogenous magnetic field with a strength of approximately $900 \mathrm{mT}$ is applied using two NdFeB permanent magnets (Supermagnete, Switzerland) mounted on a support that can be readily removed. The orientation of the magnetic field is perpendicular to the propagation direction of the probe laser beam $(\mathrm{HeNe}, \lambda=633 \mathrm{~nm})$. The polarization of the beam is oriented at a $\pi / 4$ angle to the magnetic field. A $\lambda / 4$ wave plate is placed after the sample with its fast axis parallel to the laser
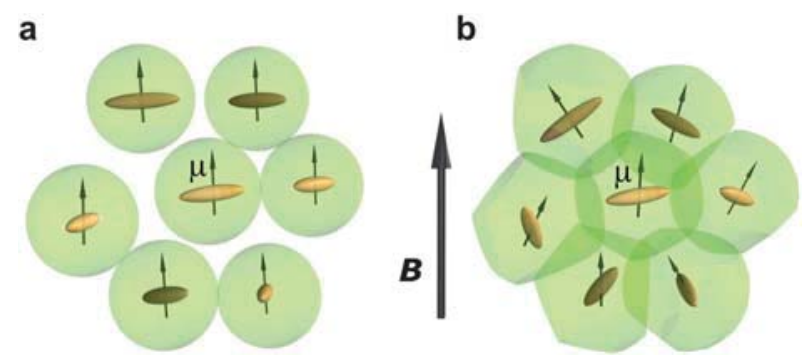

Fig. 2 Schematic diagram of the magnetic field interacting with the particles. (a) If the particles are not in contact the magnetic moments of the particles are fully aligned with the magnetic field and the ellipsoidal particle cores are randomly distributed in a plane perpendicular to the field axis. (b) For a jammed assembly elastic forces that arise from contact between neighbouring particles are competing with the magnetic torque thus resulting in a partial alignment of the magnetic moments. 
polarization direction followed by an analyser on a rotation mount at an angle $\pi / 2-\alpha$. The transmitted intensity is recorded in dependence of $\alpha$ using a calibrated power meter. To account for possible strain birefringence within the glass capillary, we normalize the measurement using a liquid dispersion of randomly oriented particles. For small $\alpha$, the relation between the optical phase shift and the transmitted intensity takes the following quadratic form: $\delta^{2} / 4+\alpha \delta-\alpha^{2} I_{\mathrm{n}}=0$, where $I_{\mathrm{n}}$ is the intensity of the transmitted light normalized by its value for randomly oriented particles. ${ }^{21}$

Each of our experiments consists of a temperature quench at a selected $\phi_{\text {eff }}$ together with the application of a homogeneous magnetic field of $900 \mathrm{mT}$. We perform "zero-field-cooling" experiments (ZFC) in which the field is switched on after the quench as well as "field-cooling" experiments (FC) wherein the field is switched on before. Additionally, for both type of experiments, we probe the relaxation of the order parameter after the field is removed. Fig. 3 shows the measured phase shifts as a function of time for a cycle of ZFC and FC experiments with a sample in a liquid state at $\phi_{\text {eff }}=0.46$ (black symbols). The response of the particles to the application of a magnetic field is constant in time and equal to $\delta=0.08$ radian in both experiments. Additionally, the phase shift decays to zero as soon as the field is removed, indicating a complete relaxation of the orientational order of the system. The field strength, $\mu B / k_{\mathrm{B}} T \approx 200$, is sufficient to fully align the magnetic moments of the hematite cores along the field direction. ${ }^{19}$ Therefore, the maximum phase shift is a measure of the saturated order parameter $S_{2}=-1 / 2$, corresponding to the long axis of the hematite cores aligned in a plane perpendicular to the applied magnetic field (Fig. 2a). ${ }^{22}$

Fig. 3 shows how the ability of the particles to align perpendicular to the applied magnetic field is hindered when the quench drives the formation of an elastic solid at $\phi_{\mathrm{eff}}=0.8$ (red symbols). The magnetic torque acting on individual particles now competes with elastic forces from neighbouring particles and the saturated
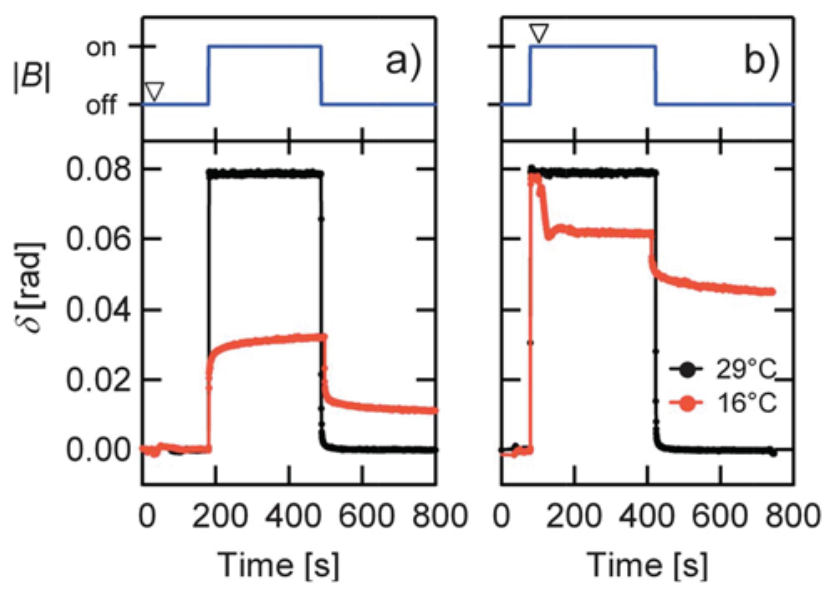

Fig. 3 Optical phase shift measurements to determine the orientation order parameter $\delta \propto\left|S_{2}\right|$. Data shown for temperature quenches from 30 ${ }^{\circ} \mathrm{C}$ to $29^{\circ} \mathrm{C}$ (red open circles, $\phi_{\text {eff }}=0.46$ ) and from $30^{\circ} \mathrm{C}$ to $16{ }^{\circ} \mathrm{C}$ (black filled circles, $\phi_{\text {eff }}=0.80$ ). (a) Zero-field-cooling experiment and (b) fieldcooling experiment. Upper panels schematically illustrate the time dependence of the external magnetic field (blue lines). Triangles indicate time when the sample is quenched to the lower temperature. value of $S_{2}$ is not reached anymore (Fig. 2b). Fig. 4 summarizes our experimental results for volume fractions ranging from $\phi_{\mathrm{eff}}=$ 0.4 to 0.9 . For clarity, we plot the absolute value of the order parameter, where the saturated value of the order parameter $\left|S_{2}\right|=1 / 2$ corresponds to the phase shift measured with liquid samples. We readily identify the onset of elasticity at a volume fraction of $\phi \approx 0.6$ in agreement with the oscillatory shear experiments. Interestingly, in both cases, the order parameter after removal of the magnetic field does not fully decay to zero. This is due to the fact that the local deformations arising from the rotation response of the particles are not elastic, but predominantly dissipative. A substantial rotation of the trapped particles magnetic moment occurs only when magnetic torque on the particle $\vec{\mu} \times \vec{B}$ exceeds a yielding threshold. Due to the complex trapping geometry with multiple contact points a purely elastic deformation, without yielding, can only lead to small angular displacements. For simplicity, in the following discussion, we neglect this contribution to the order parameter.

\section{Discussion}

In jammed colloidal or granular materials, frustrated forces and random energy landscapes are often used to model the dynamical processes at play. ${ }^{1,23}$ In these models the motion of individual particles or atoms can open free volume for others to move in, ${ }^{24}$ a process known as facilitation. ${ }^{23}$ Rotational motion however does not require substantial particle displacements or the creation of free volume. Thus in the following we argue that the resistance of our particles to orient in the presence of an external magnetic field is directly related to the local elastic trapping forces acting on the particles. For a given state point $\phi_{\text {eff, we }}$ assume that one subset of the particles $1-x^{\prime}$ is trapped elastically while the other set of particles $x^{\prime}$ is mobile and can rotate freely by Brownian motion. The strong magnetic interaction allows us to access a second, much higher scale of torque. We

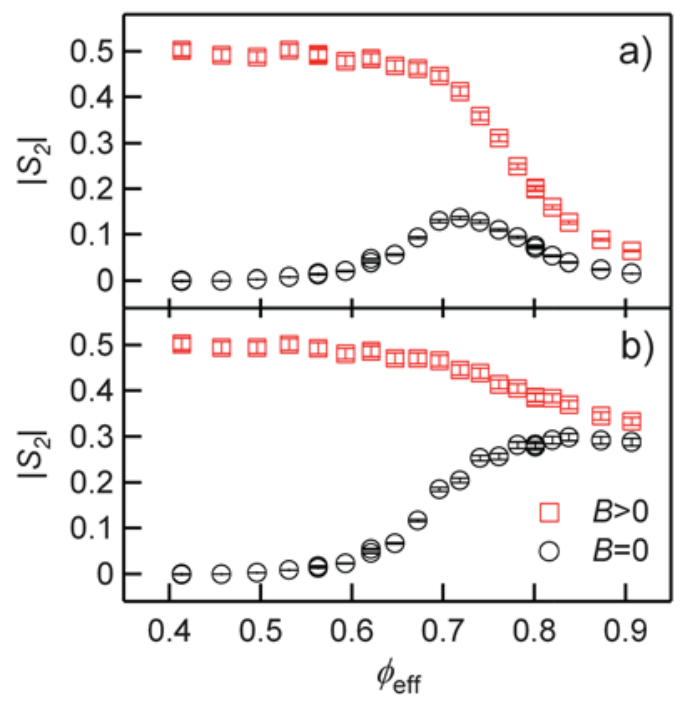

Fig. 4 Volume fraction dependence of the order parameter $\left|S_{2}\right|$. (a) Zerofield-cooling experiments and (b) field-cooling experiments. Square symbols represent $\left|S_{2}\right|$ after the magnetic field is applied $(B>0)$ and circles after the field is removed $(B=0)$. 
then denote as $x$ the fraction of particles that can be oriented by the magnetic field $\left(x>x^{\prime}\right)$.

For a zero-field-cooling experiment, the order parameter in the presence of the field is given by $\left|S_{2}\right|=x / 2$. Upon removal of the field, the order parameter drops to $\left|S_{2}\right|=\left(x-x^{\prime}\right) / 2$. Our experiment thus provides directly the fraction $x-x^{\prime}$ of trapped particles that have been involved in the yielding process. Interestingly, as a function of volume fraction, $x-x^{\prime}$ displays a clear peak around $\phi_{\text {eff }}=0.72$. While initially the fraction of elastically trapped particles increases and yielding becomes more important, above $\phi_{\text {eff }}=0.72$, the maximal elastic restoring torque starts to exceed the magnetic torque.

In the field-cooling experiments, an additional term has to be included in order to account for the observed asymptotic value of $\left|S_{2}\right| \rightarrow 0.3$. We associate this loss of order with the fraction $1-x$ of most strongly trapped particles. Initially, the particles are aligned perpendicular to the magnetic field and subsequently the sample is quenched to the desired $\phi_{\text {eff }}$. Considering a single particle, unbalanced forces that arise from contact between neighbouring particles at the liquid-solid transition give rise to a mechanical torque (Fig. 2b). This torque eventually grows larger than the magnetic torque that would otherwise hold the particle in place, and causes the drop of the order parameter in the experiments. The observed value $\left|S_{2}\right| \rightarrow 0.3$ corresponds to a random reorientation over an angular range of about $\pm 50^{\circ}$ out of plane. ${ }^{22}$ The order parameter of the system thus can be expressed as $2\left|S_{2}\right|=x+(3 / 5)(1-x)$ when the magnetic field is present, and $2\left|S_{2}\right|=\left(x-x^{\prime}\right)+(3 / 5)(1-x)$ once it is removed.

Following the simple arguments outlined above we can extract a quantitative measure of the different subpopulations of particles. The volume fraction dependence of $x$ and $x^{\prime}$ obtained using the experimental values of $\left|S_{2}\right|$ is presented in Fig. 5. The agreement between the results extracted from the zero-field-cooling (triangles) and from the field-cooling experiments (circles) underlines the consistency of our arguments.

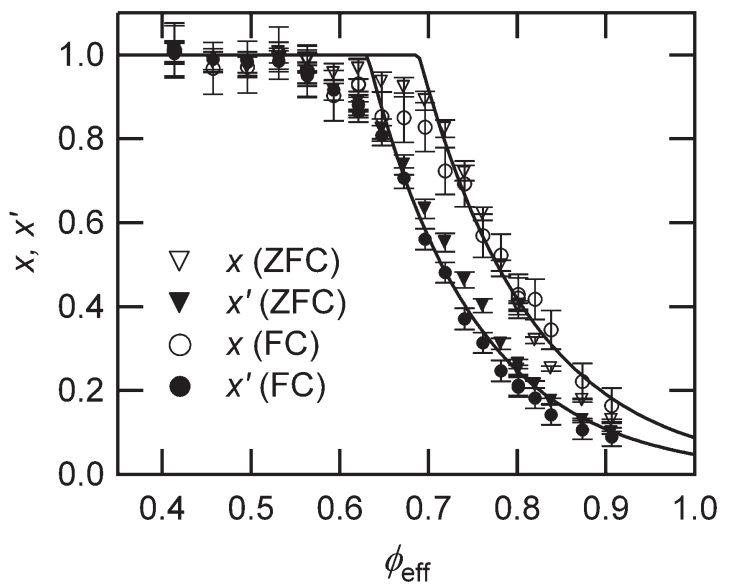

Fig. 5 Fraction of particles able to rotate in the jammed state. Full symbols: $x^{\prime}$ denotes the fraction that can rotate freely by Brownian motion. Open symbols: $x$ denotes the larger fraction able to reorient under the applied field. Independent datasets are extracted from the zerofield-cooling and the field-cooling measurements of the order parameter $\left|S_{2}\right|$. Solid lines represent exponential fits with $x^{\prime}, x=\exp \left(-\left(\phi-\phi^{*}\right) / \nu\right)$; for $\phi \geq \phi^{*}$.
The increasing degree of trapping in the arrested state becomes apparent with the $\phi$-dependence of $x$ and $x^{\prime}$. The fraction $x^{\prime}$ of the mobile particles decreases as the volume fraction is raised above the jamming transition. The decay of $x^{\prime}$ is well captured by the exponential function $x^{\prime}=\exp \left(-\left(\phi-\phi^{*}\right) / \nu\right)$; for $\phi \geq \phi^{*}$, with $\phi^{*}=0.65$ and $\nu=0.12$. We note that this value, within the experimental uncertainty, matches the jamming transition of hard spheres at $\phi \cong 0.64$. Interestingly, the decay of the fraction of the particles $x$ that reorient in the presence of the magnetic torque shows a very similar decay. We find again an exponential decrease with $\nu=0.12$ but now $\phi^{*}=0.69$.

\section{Conclusions}

With our experiments we gain the ability to measure directly trapping forces of deeply jammed colloidal particles in three dimensions. The consistent results drawn from the field-cooling and zero-field-cooling experiments indicate that local deformations arising from the rotation response of the particles are indeed predominately dissipative. Interestingly, both the fraction of mobile particles $x^{\prime}$ and the fraction $x$, able to orient in the magnetic field, decay exponentially with volume fraction. The observed similarities for these very different torques and energy scales may indicate a very similar relaxation mechanism in both cases. Variants of the experiment derived in this work may provide an even more detailed look inside jammed solids in the future. Application of a variable strength, rotating field will give direct access to the local linear and non-linear friction torque related to the yielding process described above. ${ }^{25}$ Oscillating fields could act as a magnetic shaker and provide a mechanism to inject energy in the dispersion thus raising the effective temperature, similar to electromagnetically driven granular media. ${ }^{26} \mathrm{We}$ thus expect such experiments on three-dimensional jammed solids of magneto-responsive particles to provide a wealth of quantitative data that could pave the way for a more detailed comparison of simulation, theory and experiment, something that is largely lacking to date.

\section{Acknowledgements}

Financial support from the Swiss National Science Foundation (projects no. 200021-132736, 200020-126772 and 200020-117762) and the Adolphe Merkle Foundation is gratefully acknowledged. We thank Peter Schurtenberger, Joe Brader, Veronique Trappe, Clayton Lapointe, Mathias Sperl and Holger Stark for illuminating discussions and valuable comments.

\section{Notes and references}

1 L. Berthier, Dynamical Heterogeneities in Glasses, Colloids, and Granular Media, Oxford University Press, Oxford, New York, 2011.

2 T. G. Mason, J. Bibette and D. A. Weitz, Phys. Rev. Lett., 1995, 75, 2051-2054.

3 J. F. Brady, J. Chem. Phys., 1993, 99, 567-581.

4 W. Richtering, H. Senff, C. Norhausen, A. Weiss and M. Ballauff, Langmuir, 1999, 15, 102-106.

5 F. Scheffold, P. Diaz-Leyva, M. Reufer, N. Ben Braham, I. Lynch and J. L. Harden, Phys. Rev. Lett., 2010, 104, 128304.

6 J. Mattsson, H. M. Wyss, A. Fernandez-Nieves, K. Miyazaki, Z. B. Hu, D. R. Reichman and D. A. Weitz, Nature, 2009, 462, 83-86.

7 T. S. Majmudar, M. Sperl, S. Luding and R. P. Behringer, Phys. Rev. Lett., 2007, 98, 058001 . 
8 S. Torquato, T. M. Truskett and P. G. Debenedetti, Phys. Rev. Lett., 2000, 84, 2064-2067.

9 Z. Zeravcic, N. Xu, A. J. Liu, S. R. Nagel and W. van Saarloos, Europhys. Lett., 2009, 87, 26001.

10 S. Torquato, A. Donev, I. Cisse, D. Sachs, E. Variano, F. H. Stillinger, R. Connelly and P. M. Chaikin, Science, 2004, 303, 990-993.

11 C. De Michele, R. Schilling and F. Sciortino, Phys. Rev. Lett., 2007, 98, 265702.

12 P. Pfleiderer, K. Milinkovic and T. Schilling, Europhys. Lett., 2008, 84, 49901.

13 H. N. Lee, K. Paeng, S. F. Swallen and M. D. Ediger, Science, 2009, 323, 231-234

14 M. Clusel, E. I. Corwin, A. O. N. Siemens and J. Brujic, Nature, 2009, 460, 611-615.

15 C. Dagallier, H. Dietsch, P. Schurtenberger and F. Scheffold, Soft Matter, 2010, 6, 2174-2177.

16 M. Laurenti, P. Guardia, R. Contreras-Caceres, J. Perez-Juste, A. Fernandez-Barbero, E. Lopez-Cabarcos and J. Rubio-Retama, Langmuir, 2011, 27, 10484-10491.
17 J. Rubio-Retama, N. E. Zafeiropoulos, C. Serafinelli, R. RojasReyna, B. Voit, E. L. Cabarcos and M. Stamm, Langmuir, 2007, 23, 10280-10285.

18 C. D. Jones and L. A. Lyon, Macromolecules, 2000, 33, 8301-8306.

19 M. Reufer, H. Dietsch, U. Gasser, A. Hirt, A. Menzel and P. Schurtenberger, J. Phys. Chem. B, 2010, 114, 4763-4769.

20 H. A. Stuart and A. Peterlin, J. Polym. Sci., 1950, 5, 551-563.

21 R. Piazza, V. Degiorgio and T. Bellini, Opt. Commun., 1986, 58, 400404.

22 P. G. de Gennes and J. Prost, The Physics of Liquid Crystals, Clarendon Press; Oxford University Press, Oxford New York, 2nd edn, 1993.

23 D. Chandler and J. P. Garrahan, Annu. Rev. Phys. Chem., 2010, 61, 191-217.

24 K. A. Dawson, Curr. Opin. Colloid Interface Sci., 2002, 7, 218-227.

25 N. Cappallo, C. Lapointe, D. H. Reich and R. L. Leheny, Phys. Rev. E: Stat., Nonlinear, Soft Matter Phys., 2007, 76, 031505.

26 A. Snezhko, I. S. Aranson and W. K. Kwok, Phys. Rev. Lett., 2005, 94, 108002 . 